\title{
A 12-year-old Girl With an Extraosseous Chondrosarcoma
}

\author{
Kiersten Norby ${ }^{\mathrm{a}}$, Rabia Qaiser ${ }^{\mathrm{a}}$, Alexander M McKinney IV ${ }^{\mathrm{b}}$, Cornelius H Lam ${ }^{\mathrm{a}, \mathrm{c}}$
}

\begin{abstract}
Intracranial extraosseous chondrosarcomas are a rare entity and comprise less than $0.15 \%$ of all intracranial neoplasms.A report of a pediatric extraosseous chondrosarcoma with clinical, pathologic and radiographic findings.A twelve year old girl with a left parietal heterogeneously enhancing mass, confirmed by pathology to be an extraosseous chondrosarcoma. The imaging in this case revealed no restricted diffusion on diffusion-weighted imaging (DWI) but rather showed hyperintensity on apparent diffusion coefficient (ADC) mapping. The high diffusivity (dark signal on DWI, hyperintensity on ADC map) demonstrated in our case has not been reported previously with extraosseous chondrosarcomas, and is important to note in order to distinguish them from other neoplasms such as a meningiomas.
\end{abstract}

Keywords: Extraosseous chondrosarcoma; Diffusion-weighted imaging; Apparent diffusion coefficient mapping; Pediatric intracranial neoplasm

\section{Case Report}

Intracranial extraosseous chondrosarcomas are a rare entity and comprise less than $0.15 \%$ of all intracranial neoplasms [1]. Our case is a twelve-year-old girl who presented with headaches, double vision and paresthesias. Her exam revealed papilledema bilaterally and left-sided blurry vision. Magnetic resonance imaging (MRI) of the head revealed a

Manuscript accepted for publication August 6, 2012

${ }^{a}$ Department of Neurosurgery, University of Minnesota, Minneapolis, MN, USA

${ }^{b}$ Department of Radiology, University of Minnesota, Minneapolis, MN, USA

${ }^{\circ}$ Corresponding author: Cornelius H. Lam, Mmc96, Department of Neurosurgery, 420 Delaware St. S.E., Minneapolis, MN 55455, USA Email: lamxx023@umn.edu

doi: http://dx.doi.org/10.4021/jnr125w
$6.5 \times 5.0 \times 7.0 \mathrm{~cm}$ left parietal mass (Fig. 1, 2). This was a heterogenously enhancing lesion which did not have reduced diffusion on diffusion-weighted imaging (DWI) but rather showed hyperintensity on apparent diffusion coefficient (ADC) mapping (Fig. 2).

The patient underwent primary resection where there was good brain-tumor differentiation however diffuse hemorrhage from the tumor limited total resection. Several weeks later the patient underwent a second resection to remove the residual tumor. Grossly, the tumor was an aggregate of pale yellow-tan focally translucent soft tissue. Microscopically, the tissue appeared as a myxoid neoplasm with relatively low cellularity. The tumor cells were relatively small with eccentric processes embedded in a myxoid matrix. Immunohistochemical stains for vimentin were strongly positive while stains for S100 protein, glial fibrillary acidic protein (GFAP), epithelial membrane antigen (EMA) and CD34 were negative. Ki-67 labeling was relatively sparse with maximal labeling of approximately five percent of cells.

To expand on the importance of the immunohistochemical stains the following offers a brief description. Vimentin is an intermediate filament found in mesenchymal tissue and is used as a marker for mesenchymal tumors. S100 is a protein involved in many critical cell activities which stains positive in most melanomas. GFAP is an intermediate filament found in astrocytes and can be used to identify CNS tumors. Ki-67 is a protein expressed in all phases of the cell cycle except G0 and can be used as a marker of cell proliferation. EMA is a large cell surface mucin glycoprotein expressed by most glandular and ductal epithelial cells; it is expressed by most adenocarcinomas. EMA may also positively stain large cell lymphomas, meningiomas and plasmacytomas. CD34 is a cell surface glycoprotein that functions as a cell-cell adhesion factor, it is normally present on bone marrow and hematopoietic cells as well as some lymphoid neoplasms, meningiomas, neurofibromas, schwannomas, and malignant nerve sheath tumors. Thus the diagnosis of extraosseous myxoid chondrosarcoma was made from the histological appearance as well as the staining pattern (Fig. 3).

Post-operatively the patient recovered well with improvement in her vision as well as her headaches. The patient will continue to be followed by Oncology as well as Neuro- 


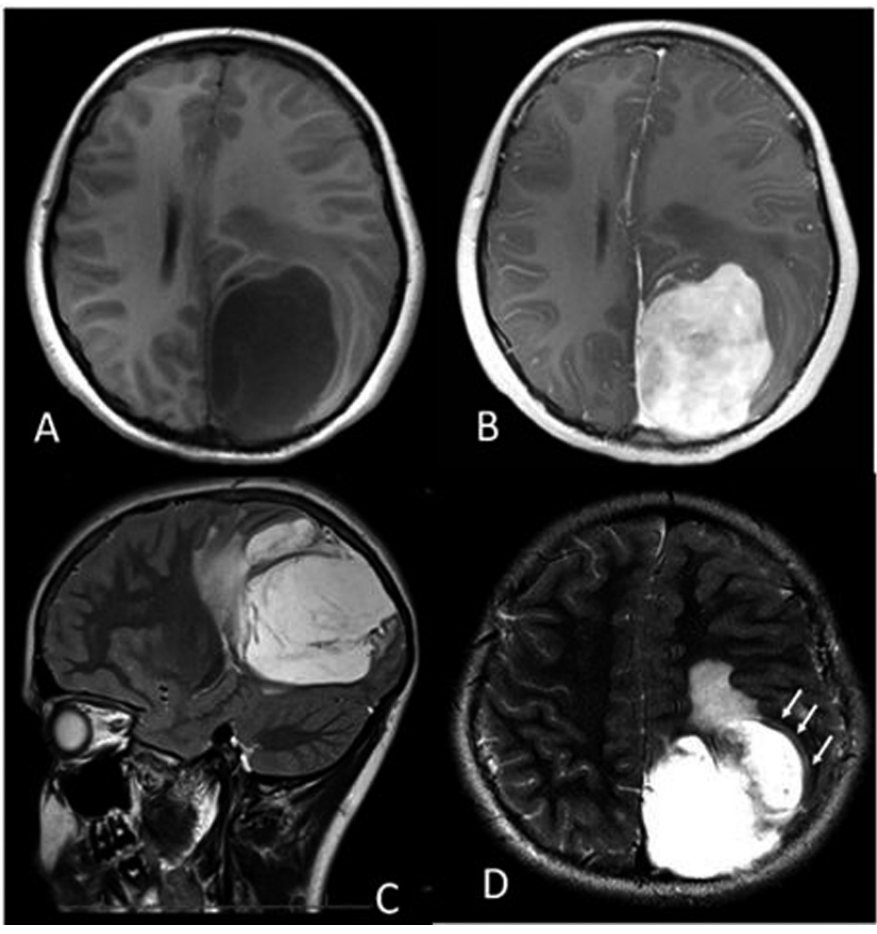

Figure 1. (A) Axial T1-weighted MRI showing the hypointense left parietal mass measuring approximately $6.5 \times 5.0 \times$ $7.0 \mathrm{~cm}$, with $1.0 \mathrm{~cm}$ midline shift. (B) Axial post contrast T1-weighted MRI showing the heterogeneous, enhancing left parietal mass. (C) Sagittal T2-weighted image showing hyperintensity of the mass, with edema anteriorly. (D) Axial T2-weighted image depicting what is likely a CSF "cleft" (arrows), suggesting that the mass is extra-axial.
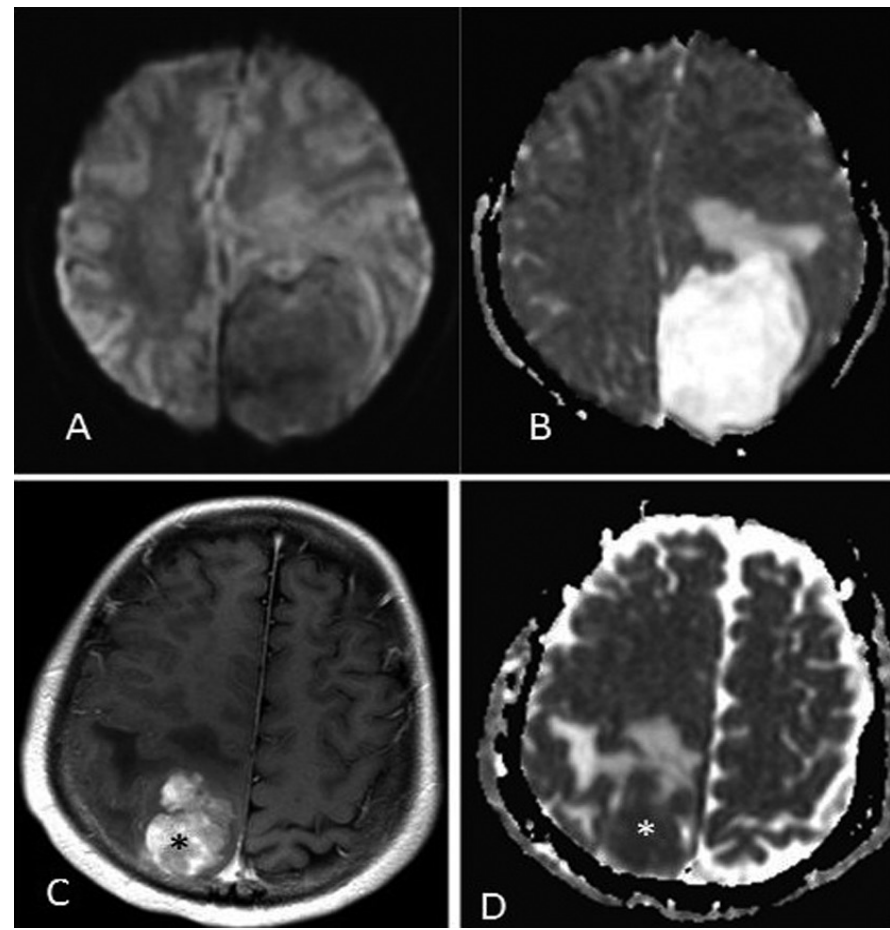

Figure 2. (A) DWI MRI showing the left parietal mass without reduced diffusion. (B) ADC MRI showing the mass with abundant surrounding vasogenic edema, and internally high diffusivity (mean $A D C=2.221 \times 10^{-3} \mathrm{~cm}^{2} / \mathrm{sec}$ ). For the purposes of comparison, a meningiosarcoma in a 17 year old is shown on post-contrast T1-weighted MRI (C), with ADC map (D) that has internally low diffusivity (asterisks, $A D C=0.677 \times 10^{-3} \mathrm{~cm}^{2} / \mathrm{sec}$ ). 


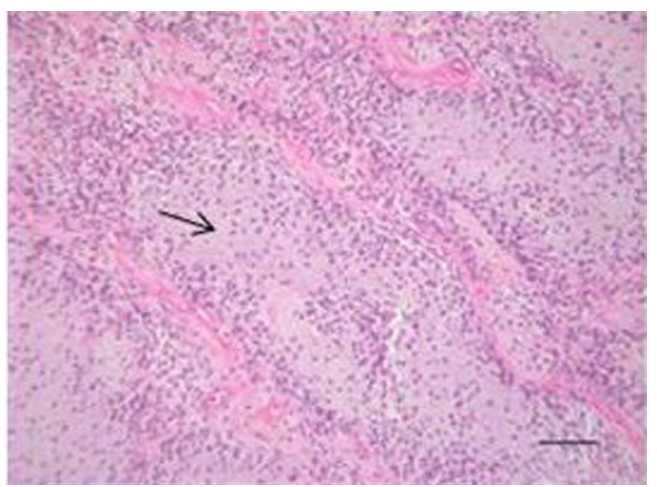

Figure 3. Hematoxylin and eosin staining showing the tumor with myxoid matrix and relatively low cellularity (Arrow showing myxoid matrix).

surgery for further management and surveillance.

\section{Discussion}

As previously mentioned, extraosseous myxoid chondrosarcomas are rare intracranial neoplasms, most often seen in adults arising from the skull base [2]. Only four cases of intracranial extraosseous chondrosarcomas have been reported in the literature and out of them only one was a pediatric case in the posterior fossa [3]. Extraosseous chondrosarcomas are typically characterized by a balanced chromosome translocation involving $\mathrm{t}(9 ; 22)(\mathrm{q} 22 ; \mathrm{q} 12)$ with resultant fusion of the genes EWSR1 and NR4A3 [4]. Microscopically extraosseous chondrosarcomas show some resemblance to embryonal cartilage, however, because of the wide morphological spectrum clearly defined histology is difficult [5].

Historically, a few theories have arisen concerning the embryological origin of the tumor which will be briefly mentioned here. First, that the tumor arises from embryonal tissue which was displaced from the base of the skull or along the paths of migrating blood vessels. Second, the tumor arises from metaplasia of fibroblasts. And finally, the tumor is formed de novo from multipotent cells of the meninges or choroid plexus [6].

The imaging in this case is particularly interesting due to the lesion's appearance on DWI and ADC mapping (Fig. 2 ). While extraosseous chondrosarcomas typically exhibit low signal on T1-weighted sequences and high signal on T2weighted sequences, as in our case (Fig. 1), their appearance on DWI has not yet been described [2]. Most "soft-tissue" (mesenchymal-origin) sarcomas have consistently demonstrated lower ADC values (represented as bright signal on DWI) with an inverse correlation between tumor cellularity and ADC values [7].

Chondrosarcomas remain a notable exception, consistently demonstrating high ADC values, i.e. "high diffusivity," based on measurements of osseous chondrosarcomas
[7]. Although the DWI characteristics of extraosseous chondrosarcomas have not yet been described, the appearance of extraosseous and osseous chondrosarcomas is quite similar on other MR sequences, and thus their DWI characteristics of high diffusivity (high ADC values) should be similar [8]. Given the avid contrast enhancement in our patient, the DWI findings could mistakenly suggest that there is not high cellularity of the extra-axial mass if chondrosarcoma was not considered in the differential diagnosis.

Extra-axial masses were considered in our case, the primary consideration being meningioma (Fig. 2). Meningiomas also exhibit avid enhancement and demonstrate variable diffusivity (ADC range $=0.45-1.80 \times 10^{-3} \mathrm{~cm}^{2} / \mathrm{sec}$. in meningiomas) [8]. However, the ADC values are lower even in benign meningiomas compared to the ADC values reported in osseous chondrosarcomas (mean ADC of $1.69-2.54 \mathrm{x}$ $10^{-3} \mathrm{~mm}^{2} / \mathrm{sec}$ ) [8]. Thus, the high diffusivity (dark signal on DWI, hyperintensity on ADC map) demonstrated in our case has not been reported previously with extraosseous chondrosarcomas, and is important to note in order to distinguish it from a meningioma.

There is no consensus on the best modality of treatment, but based on the intracranial location of extraskeletal myxoid chondrosarcomas they typically behave as a low-grade sarcoma and the treatment of choice seems to be radical resection. Extraskeletal myxoid chondrosarcomas have a five year survival rate ranging from $65 \%$ to $85 \%$. Based on a review of limited literature available, we will be following our patient with serial scans and are opting to refrain from radiation or chemotherapy given her young age.

\section{Conflict of Interest Disclosure}

The authors have no financial or other conflicts of interest to disclose.

\section{References}

1. Salcman M, Scholtz H, Kristt D, Numaguchi Y. Extraskeletal myxoid chondrosarcoma of the falx. Neurosurgery. 1992;31(2):344-348.

2. O'Brien J, Thornton J, Cawley D, Farrell M, Keohane K, Kaar G, McEvoy L, et al. Extraskeletal myxoid chondrosarcoma of the cerebellopontine angle presenting during pregnancy. Br J Neurosurg. 2008;22(3):429-432.

3. Smith TW, Davidson RI. Primary meningeal myxochondrosarcoma presenting as a cerebellar mass: case report. Neurosurgery. 1981;8(5):577-581.

4. Sjogren H, Meis-Kindblom JM, Orndal C, Bergh P, Ptaszynski K, Aman P, Kindblom LG, et al. Studies on the molecular pathogenesis of extraskeletal myxoid chondrosarcoma-cytogenetic, molecular genetic, and cDNA mi- 
croarray analyses. Am J Pathol. 2003;162(3):781-792.

5. Enzinger FM, Shiraki M. Extraskeletal myxoid chondrosarcoma. An analysis of 34 cases. Hum Pathol. 1972;3(3):421-435.

6. Wu WQ, Lapi A. Primary non-skeletal intracranial cartilaginous neoplasms: report of a chondroma and a mesenchymal chondrosarcoma. J Neurol Neurosurg Psychiatry. 1970;33(4):469-475.

7. Schnapauff D, Zeile M, Niederhagen MB, Fleige B,
Tunn PU, Hamm B, Dudeck O. Diffusion-weighted echo-planar magnetic resonance imaging for the assessment of tumor cellularity in patients with soft-tissue sarcomas. J Magn Reson Imaging. 2009;29(6):1355-1359.

8. Filippi CG, Edgar MA, Ulug AM, Prowda JC, Heier LA, Zimmerman RD. Appearance of meningiomas on diffusion-weighted images: correlating diffusion constants with histopathologic findings. AJNR Am J Neuroradiol. 2001;22(1):65-72. 